\title{
Quantum Dot-Based Aptasensor for Sensitive Protein Detection
}

\author{
Seonmi Shin, Yea Seul Kim, Ho-Jung Kang, and Sang Soo Hah* \\ Department of Chemistry and Research Institute for Basic Sciences, Kyung Hee University, Seoul 130-701, Korea \\ *E-mail:sshah@khu.ac.kr \\ Received May 18, 2010, Accepted May 26, 2010
}

Key Words: Western blot, Quantum dot, Aptasensor

Aptamers are a special class of nucleic acids (RNAs or DNAs) selected from systematic evolution of ligands by exponential enrichment process in vitro that specifically bind to a target molecule with high affinity. ${ }^{1,2}$ Emerging as alternatives to antibodies, a wide range of aptamers have been found to bind specifically to proteins. Aptamers, however, offer several advantages over antibodies, due to their relative ease of isolation and modification, tailored binding affinity, and resistance against denaturizing. Moreover, their target molecules include metal ions, small organic compounds, metabolites, proteins and even cells. Thus, aptamers have been used in many bioanalytical applications, such as for specific detection of proteins, ${ }^{2-5}$ metal ions, ${ }^{6-8}$ and small molecules, ${ }^{9,10}$ and for target-specific delivery. ${ }^{11}$ Research focused on aptamers has also exhibited promising potentials in various fields such as pharmaceutics and diagnostics.

The most popular techniques for signal production caused by aptamer-biomolecule interactions use electrochemical- and optical-detection platforms. ${ }^{1,2}$ These usually have sensitivities that allow detection of micromolar to nanomolar concentrations. In the previous studies, DNA-based aptamer-functionalized gold nanoparticles have been used for the detection of thrombin and adenosine. ${ }^{1-5,9,10}$ Aptamer-functionalized nanoparticles were synthesized by direct chemisorptions of thiolated oligonucleotides, by biotin-streptavidin interaction, or by simple adsorption. ${ }^{1-8}$

While aptamers are attractive as binding probes, quantum dots (QDs) have attracted much attention as optical probes because of their interesting optical properties. ${ }^{2,13}$ QDs are semiconducting nanoparticles and of the several available types of QDs, CdSe/ZnS QDs have recently emerged as a useful and alternative material for biomolecular labeling due to their unique optical advantages, including photostability, narrow emission bands, broad excitation spectra, and accessibility to versatile functionalization that allows site-specific targeting. ${ }^{12,13}$ The fluorescent properties of QDs are one of the key advantages for their use in specific and sensitive protein detection over currently used detection technology, ${ }^{14}$ since QDs absorb light over a broad spectral range and fluoresce at wavelengths determined by their physical sizes, producing precise and narrow spectral emissions, and QDs are extremely efficient at absorbing light and converting it to a highly stable fluorescent emission, making them up to approximately 50 times brighter than conventional organic fluorophores. ${ }^{12,13}$ This high level of brightness and excellent photostability bring the added value of high sensitivity and ruggedness. Moreover, multiple functionality can be introduced to exploit the binding cooperativity. ${ }^{15}$ Recently, antibody-conjugated QDs have been successfully used in Western blots to detect the tracer proteins. ${ }^{16}$

In the present study, we took advantage of the properties both of aptamers and QDs, generating 15-mer ssDNA thrombinbinding aptamer conjugated QDs (Scheme 1), in order to develop an alternative to the conventional Western blots.

Among a wide variety of potential biofunctional groups, we used the highly selective and strong interaction of a 15-mer ssDNA aptamer complex with thrombin $\left(K_{\mathrm{d}} \sim 2.68-450 \mathrm{nM}\right){ }^{17}$ since thrombin, a serine protease, plays important roles in the coagulation cascade, thrombosis and haemostasis and the highpicomolar range of thrombin in blood has been known to be associated with several diseases so that it has been important to assess this protein at trace level with high sensitivity. ${ }^{17}$ The affinity between DNA aptamer and thrombin has been known to be comparable to that of protein-antibody $\left(K_{\mathrm{d}} \sim 10^{-5}-10^{-12}\right.$ $\mathrm{M}){ }^{18}$ Therefore, the use of such an interaction could provide a simple, selective and sensitive method that would overcome the limitation of conventional antibody-based protein labeling and also could be applied across a wide range of applications (see Figure 1 for the advantages of our approach over conventional protein detection methods).

To check the sensitivity of aptamer-modified QDs against thrombin, we compared our QD-based aptasensor system with

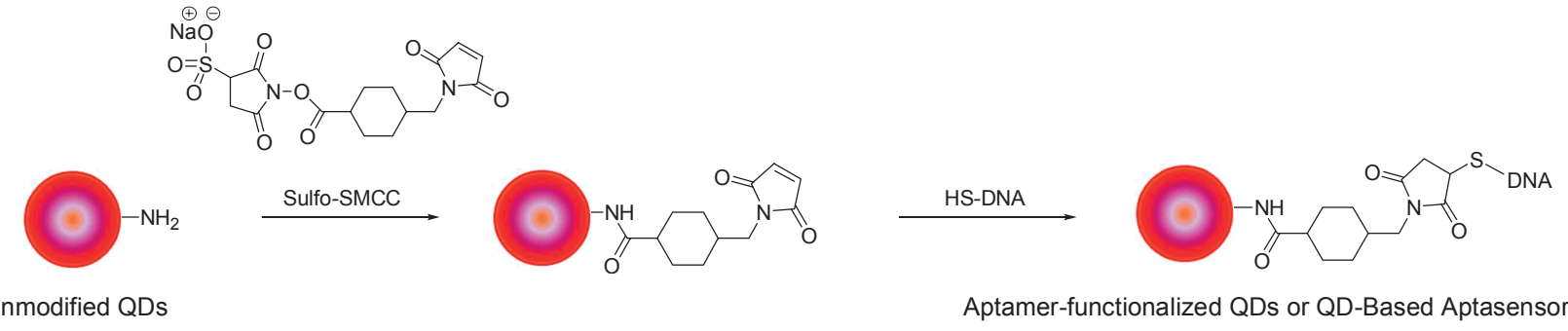

Scheme 1. Scheme of thrombin aptamer conjugation on quantum dots (QDs) 


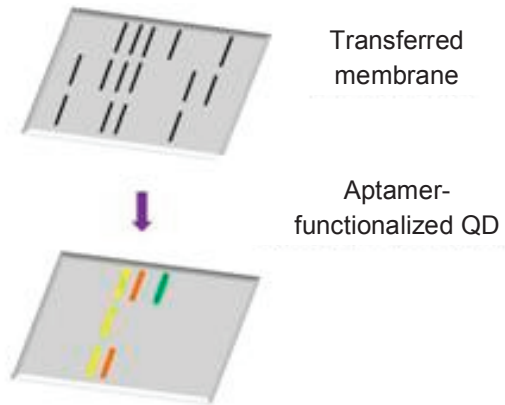
Advantages including:
1. time-saving
2. no requirement for antibodies
3. no need for ${ }^{32} \mathrm{P}$
4. multiplexing detection when using more than 2 kinds of QD-Aptamers
5. ease to reuse
6. almost-not-limited applicabilities

Figure 1. Schematic illustration of protein detection using aptamerfunctionalized QDs and possible advantages of our method over the conventional protein detection methods such as Western blots are listed.

the conventional Coomassie blue staining method (BIO-RAD, CA). As shown in Figure 2, the indicated amounts of thrombin were loaded onto a $15 \%$ SDS-PAGE gel. The proteins and molecular weight markers (Sun Genetics, Korea) were separated according to size, followed by transfer to a Protran nitrocellulose membrane (Whatman, NJ), using a Mini Trans-Blot Cell electroblotter (Bio-Rad, CA). Subsequently, the membrane was incubated in Ponceau S solution for $5 \mathrm{~min}$ at room temperature. The membrane was rinsed three times with $0.1 \%$ phosphate-buffered saline Tween-20 (PBST) and incubated with aptamer-functionalized QDs or QD-based aptasensor containing $0.1 \% \mathrm{PBST}$ for $5 \mathrm{~h}$ at room temperature. After incubation, the membrane was rinsed three times with double-distilled water. Images were captured using a BIO-RAD ChemiDoc XRS system with UV transillumination mode.

Although reagents and gel imaging system need to be further optimized in future works for higher sensitivity and lower background signal, our QD system could successfully detect only thrombin as low as $300 \mathrm{ng}$ with 2-h incubation time, and the obtained signals were by large linear over a range of 0.300 $\mu \mathrm{g}$ to $1.5 \mu \mathrm{g}$ (Figure 2). However, with the same incubation time, the blot that had been probed with the anti-thrombin antibody did not generate any visible signal. Detectable bands appeared only on the blot with overnight incubation. This clearly demonstrates that our QD-based blotting system exhibited an even better signal with similar linearity as Coomasie blue staining method, although more sophisticated image acquisition and analysis methods are likely to improve the results in the present study. Interestingly, our QD-based aptasensor could discriminate two isoforms of thrombin. We also found that our QD-based aptasensor could be stored in a buffer at $4{ }^{\circ} \mathrm{C}$ with minimal loss of signal for imaging at a later date.

In theory, QD-based aptasensor imaging is flexible and can be done at several levels of sophistication. With a gel imaging or documentation workstation, detection varies with the illumination source and the installed filters on that particular model

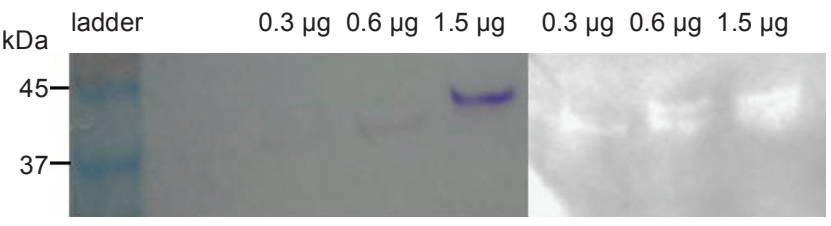

Figure 2. Results of QD-based Western blot analysis for thrombin detection. At right, a result from the commercially available Coomassie blue staining (BIO-RAD, CA) is shown in comparison with our QD system.

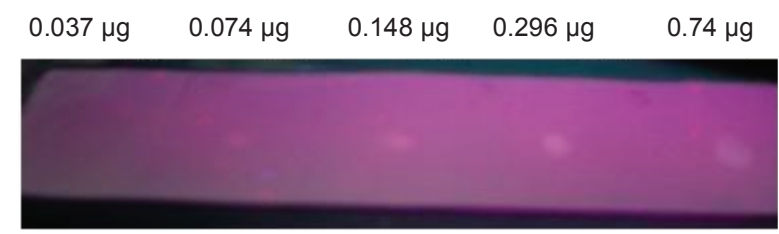

Figure 3. Results of QD-based dot-blotting analysis for thrombin detection.

of the instrument, since most gel imagers have a source sufficient to excite QD conjugates, such as an epi-UV illuminator. At a minimum, for example, a hand-held blue or ultraviolet light source and a high-quality color digital camera can be used to illuminate the blot and capture images of the fluorescent protein bands, as demonstrated in Figure 3. The indicated amounts of thrombin were dot-blotted onto a Protran nitrocellulose membrane (Whatman, NJ), and the membrane was subsequently incubated in Ponceau $\mathrm{S}$ solution for $5 \mathrm{~min}$ at room temperature. The membrane was rinsed three times with $0.1 \%$ phosphatebuffered saline Tween-20 (PBST) and incubated with aptamerfunctionalized QDs or QD-based aptasensor containing 0.1\% PBST for $5 \mathrm{~h}$ at room temperature. After incubation, the membrane was rinsed three times with double-distilled water. Images were captured using a hand-held ultraviolet light source and a color digital camera and capture images of the fluorescent protein spots. Very importantly, our aptasensor was found to have a detection limit as low as $2 \mathrm{nmol}$ or $74 \mathrm{ng}$ of thrombin. The minimum amount of protein that can be detected with our method is thought to be a function of aptamer affinity and the source of background fluorescence in the image, such as the nonspecific binding of the given aptamer and the auto-fluorescence of the blotting membrane, considering several protein-antibody combinations in the literature that have shown sensitivities to the low pictogram level with a 100 -fold linear concentration range.

The benefits of QDs in biological applications are being appreciated more than ever and hence, scientists are introducing QDs in biological applications. Our DNA aptamer conjugated QDs Western blotting system dramatically reduced the number of steps required, as well as the time and cost involved, compared with the traditional system. In addition, the specificity and signal strength were similar to or better than those with the traditional methods. Moreover, we obtained a linear signal over a range of $0.300 \mu \mathrm{g}$ to $1.5 \mu \mathrm{g}$ of thrombin making quantitative analysis of Western blots easier and more reliable. It 
should be also noted that in principle, owing to the optical properties of QDs and a wide versatility of aptamers for selection, our system can harness the high brightness, extreme stability and reusability to quantitatively detect aptamer-recognizable proteins, and due to the versatility of aptamers, this method can be applied to any protein, nucleic acids, or even small molecules and metal ions. Furthermore, sensitive multiplex detection for several proteins on a single blot can be achieved by our new method, which thus may be able to facilitate and simplify the routinely used protein detection procedure, and make a variety of proteomics analysis possible, including analyses of differential expression and post-translational modifications such as glycosylation and phosphorylation or dephosphorylation activity.

To summarize, we employed QD-conjugated DNA aptamer interactions with thrombin, leading to development of an alternative to the conventional Western blots. The aptasensor was found to have a detection limit as low as $2 \mathrm{nmol}$ or $74 \mathrm{ng}$ of thrombin, when using a hand-held ultraviolet light source and a color digital camera to illuminate the blot and capture images of the fluorescent protein bands or dots. Since DNA aptamers are superior to RNA aptamers from the stability point of view and they offer several advantages over antibodies, our study brings the power of QD fluorescence technology to a workhorse application in proteomics. In principle, owing to the remarkable optical properties of QDs and a wide versatility of aptamers for selection, our system can be used for multiplex detection of target proteins in the human serum sample. Such a QD-based aptasensor may be able to facilitate and simplify the routinely used protein detection procedure, and provide a promising strategy for screening biomarkers at ultratrace levels in the complex matrices.

\section{Experimental Section}

Reagents were obtained from commercial suppliers and were used without further purification, and double-distilled water was used for all experiments. QDs with emission maxima of $655 \mathrm{~nm}$ and modified with PEG and amino groups were obtained from Invitrogen (Carlsbad, CA). QD concentrations were measured by optical absorbance, using extinction coeeficients provided by the supplier. Cross-linker used was sulfosuccinimidyl 4-( $N$-maleimidomethyl)cyclohexane-1-carboxylate (sulfo-SMCC, Sigma). HPLC-purified ssDNA-based aptamers (5'-HS- $\left(\mathrm{CH}_{2}\right)_{6}$-GGT TGG TGT GGT TGG-3') for specific binding to thrombin were purchased from Bioneer (Daejeon, Korea), and thrombin (human $\alpha$-thrombin) was from SigmaAldrich.

Amino-modified QDs were conjugated to thiol-containing ssDNA aptamers using sulfo-SMCC cross-linker (Scheme 1) according to the literature. ${ }^{19}$ In brief, QDs, Qdot ${ }^{\circledR} 655$ ITK $^{\mathrm{TM}}$ amino (PEG) quantum dots (Invitrogen, CA) were resuspended in $50 \mathrm{mM}$ sodium phosphate, $150 \mathrm{mM}$ sodium chloride, $\mathrm{pH} 7.2$.
Cross-linker (100-fold excess) was added to QDs and allowed to react for $1 \mathrm{~h}$. Samples were filtered on a NAP-5 gravity column (to remove excess cross-linker) into similar buffer supplemented with $10 \mathrm{mM}$ EDTA. 5'-Thiol-containing ssDNA aptamer was added to filtered QDs and allowed to react overnight at $4{ }^{\circ} \mathrm{C}$. Using three Amicon filters, product was filtered twice with Dulbecco's phosphate-buffered saline (PBS), twice with a high salt buffer (1.0 M sodium chloride, $100 \mathrm{mM}$ sodium citrate, $\mathrm{pH}$ 7.2), and twice again with PBS. High salt washes were required to remove electrostatically bound DNA, which was not removed with PBS washes alone. The prepared QDs were stable and maintained strong fluorescence in doubledistilled water.

Acknowledgments. We are grateful to Professor Dong-Eun Kim for his helpful advice. This work was supported by Basic Science Program through the National Research Foundation of Korea (KRF) funded by the Ministry of Education, Science and Technology (MEST) (No. 2010-0015218).

\section{References}

1. Nimjee, S. M.; Rusconi, C. P.; Sullenger, B. A. Annu. Rev. Med. 2005, 56, 555.

2. Pavlov, V.; Xiao, Y.; Shlyahovsky, B.; Willner, I. J. Am. Chem. Soc. 2004, 126, 11768.

3. Ho, H. A.; Leclerc, M. J. Am. Chem. Soc. 2004, 126, 1384.

4. Xiao, Y.; Lubin, A. A.; Heeger, A. J.; Plaxco, K. W. Angew. Chem. Int. Ed. 2005, 44, 5456.

5. Balamurugan, S.; Obubuafo, A.; Soper, S. A.; McCarley, R. L.; Spivak, D. A. Langmuir 2006, 22, 6446.

6. Wang, L.; Liu, X.; Hu, X.; Song, S.; Fan, C. Chem. Commun. 2006, 3780.

7. He, F.; Tang, Y.; Wang, S.; Li, Y.; Zhu, D. J. Am. Chem. Soc. 2005, $127,12343$.

8. Ueyama, H.; Takagi, M.; Takenaka, S. J. Am. Chem. Soc. 2002, $124,14286$.

9. Sankaran, N. B.; Nishizawa, S.; Seino, T.; Yoshimoto, K.; Teramae, N. Angew. Chem. Int. Ed. 2006, 45, 1563.

10. Liu, J.; Lu, Y. Angew. Chem. Int. Ed. 2006, 45, 90.

11. Bagalkot, V.; Farokhzad, O. C.; Langer, R.; Jon, S. Angew. Chem. Int. Ed. 2006, 45, 8149 .

12. Alivisatos, A. P.; Gu, W.; Larabell, C. Annu. Rev. Biomed. Eng. 2005, 7, 55.

13. Alivisatos, A. P. Nat. Biotechnol. 2004, 22, 47.

14. Michalet, X.; Pinaud, F. F.; Bentolila, L. A.; Tsay, J. M.; Doose, S.; Li, J. J.; Sundaresan, G.; Wu, A. M.; Gambhir, S. S.; Weiss, S. Science 2005, 307, 538.

15. Suzuki, M.; Husimi, Y.; Komatsu, H.; Suzuki, K.; Douglas, K. T. J. Am. Chem. Soc. 2008, 130, 5720.

16. Bakalova, R.; Zhelev, Z.; Ohba, H.; Baba, Y. J. Am. Chem. Soc. 2005, 127, 9328 .

17. Li, J. J.; Fang, X.; Tan, W. Biochem. Biophys. Res. Commun. 2002, 292,31

18. Guignet, E. G.; Hovius, R.; Vogel, H. Nat. Biotechnol. 2004, 22, 440 .

19. Derfus, A. M.; Chen, A. A.; Min, D.-H.; Ruoslahti, E.; Bhatia, S. N. Bioconjug. Chem. 2007, 18, 1391. 